\title{
Enhanced hydrogen adsorption in boron substituted carbon nanospaces
}

\author{
L. Firlej, ${ }^{1,2}$ Sz. Roszak, ${ }^{3}$ B. Kuchta, ${ }^{2,4, a)}$ P. Pfeifer, ${ }^{2}$ and Carlos Wexler ${ }^{2}$ \\ ${ }^{1}$ LCVN, University of Montpellier 2, 34095 Montpellier, France \\ ${ }^{2}$ Department of Physics and Astronomy, University of Missouri, Columbia, Missouri 65211, USA \\ ${ }^{3}$ Institut of Physical and Theoretical Chemistry, Wroclaw University of Technology, 50370 Wroclaw, Poland \\ ${ }^{4}$ Labortoire Chimie Provence, University of Aix-Marseille 1, 13397 Marseille, France
}

(Received 14 July 2009; accepted 30 September 2009; published online 23 October 2009)

\begin{abstract}
Activated carbons are one of promising groups of materials for reversible storage of hydrogen by physisorption. However, the heat of hydrogen adsorption in such materials is relatively low, in the range of about $4-8 \mathrm{~kJ} / \mathrm{mol}$, which limits the total amount of hydrogen adsorbed at $P=100$ bar to $\sim 2$ wt $\%$ at room temperature and $\sim 8$ wt $\%$ at $77 \mathrm{~K}$. To improve the sorption characteristics the adsorbing surfaces must be modified either by substitution of some atoms in the all-carbon skeleton by other elements, or by doping/intercalation with other species. In this letter we present ab initio calculations and Monte Carlo simulations showing that substitution of 5\%-10\% of atoms in a nanoporous carbon by boron atoms results in significant increases in the adsorption energy (up to $10-13.5 \mathrm{~kJ} / \mathrm{mol})$ and storage capacity $(\sim 5 \mathrm{wt} \%$ at $298 \mathrm{~K}, 100$ bar) with a $97 \%$ delivery rate. (C) 2009 American Institute of Physics. [doi:10.1063/1.3251788]
\end{abstract}

Hydrogen is widely considered an essential part of our energy future despite the substantial difficulties derived from its low volumetric energy density. The development of a suitable material for reversible storage of hydrogen still remains a "grand challenge,", in particular for vehicular applications. ${ }^{1}$ Although some materials are potentially attractive, even the most promising candidates, nanoporous activated carbons, ${ }^{3-6}$ have yet to meet the U.S. Department of Energy (DOE) 2010 targets $\left(0.045 \mathrm{~kg} \mathrm{H}_{2} / \mathrm{kg}\right.$ system, $28 \mathrm{~kg}$ $\mathrm{H}_{2} / \mathrm{m}^{3}$ system at room temperature for light-duty vehicles ${ }^{7}$ ). Recent achievement in engineering carbons nanospaces resulted in preparation of very high surface area materials $\left(>3000 \mathrm{~m}^{2} / \mathrm{g}\right)$ performing exceedingly well at cryogenic temperature $\left(\sim 0.1 \mathrm{~kg} \mathrm{H}_{2} / \mathrm{kg}\right.$ system at $\left.P=100 \mathrm{bar}^{6,8}\right)$. However, the low heat of hydrogen physisorption on carbons (4-8 kJ/mol) results in low storage capacities at room temperature $\left(\sim 0.02 \mathrm{~kg} \mathrm{H}_{2} / \mathrm{kg}\right.$ system at $P=100$ bar $){ }^{5,6}$ The challenge is, thus, to find ways to increase the interaction of hydrogen and a carbon substrate.

Boron-doped carbons are widely conjectured to be the suitable materials for hydrogen storage. It is believed that boron doping raises the binding energy to levels that would enable room temperature storage at moderate $(<100$ bar $)$ pressures. This increase in the binding energy appears to be caused by boron acting as a $p$-type dopant, introducing electron deficiency in the graphite layer planes, thus lowering the Fermi level and increasing the surface polarizability. ${ }^{9,10}$ It is also possible that a partial charge transfer from the occupied $\sigma$ orbital of $\mathrm{H}_{2}$ to the empty $p_{z}$ orbital of $\mathrm{B}$ increases the interaction energy between $\mathrm{H}_{2}$ and boron substituted carbon surface. ${ }^{11,12}$ So far, however, neither solid computational evidence (i.e., from first principles, beyond Ref. 11 for B-doped fullerenes) nor clear experimental evidence for enhanced hydrogen storage capacities of boron-doped carbons have been

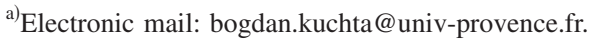

presented (to the best of our knowledge). Specifically, a major question is to what extent a single boron atom, substituted in graphitic carbon surface, creates high binding energies not only locally at the substitution site but also on nearestneighbor and perhaps even next-nearest-neighbor carbon atoms.

This paper, for the first time, presents general/generic evidence-from first-principles ( $a b$ initio) computations of adsorption potentials of boron-substituted carbons and grand canonical Monte Carlo (GCMC) simulations of hydrogen adsorption equilibria in the resulting potentials-that boron doping massively increases storage capacities, with as little as a $5 \%-10 \%$ boron concentration. The results make a strong case that it is only a matter of time until superior hydrogen storage capacities on boron-doped carbons will be observed experimentally.

The interaction of $\mathrm{H}_{2}$ with modified carbon nanostructures is to a large extent controlled by dispersion terms and its evaluation requires the proper treatment of the correlation energy. It can be achieved using one of two available basic methods, Møller-Plesset ${ }^{13}$ or coupled cluster ${ }^{14}$ approaches (the latter is usually considered to be of higher precision). In the present study we used the Møller-Plesset approach for two reasons. First, the available data ${ }^{15,16}$ indicate that the adsorption energy calculated within both approaches differs by a few percent only while the geometry remains practically unchanged. Second, the Møller-Plesset calculations are less computational time consuming than the coupled clusters method while retaining sufficient precision for our procedure, where a reduction in precision is unavoidable when the potential energy surfaces are being converted into interatomic potentials for Monte Carlo simulations.

In view of these considerations, first ab initio calculations were performed, to get the energy of interactions between $\mathrm{H}_{2}$ and boron atoms substituted in the graphene matrix. The interactions of hydrogen molecule with boron 
modified graphene surface were modeled through interactions of $\mathrm{H}_{2}$ with pyrene or its B-derivative. The external carbon frame of pyrene was kept frozen in B-derivative to better represent the real modified graphite material. The optimized boron position preserves the planar structure. The $a b$ initio studies were performed applying the second order Møller-Plesset level of theory utilizing restricted open Hartree-Fock wave function. The present calculations were performed using the effective core potential SBJKJC VDZ basis $\operatorname{set}^{17}$ embedded by polarization functions $d$ for boron and carbon and $p$ function for hydrogen. ${ }^{18}$ All the calculations were carried out using the GAUSSIAN 03 suite of codes. ${ }^{19}$ The $\mathrm{H}_{2}$ molecule forms two perpendicular complexes with pyrene surface. The minimum energy structures were determined for hydrogen interacting with $\mathrm{B}$-center $\left[\Delta E=7.8 \mathrm{~kJ} / \mathrm{mol}, \quad R\left(\mathrm{~B}-\mathrm{H}_{2}\right)=3.12 \AA\right]$ and $\mathrm{C}$-center $\left(\Delta E=5.6 \mathrm{~kJ} / \mathrm{mol}, R\left(\mathrm{C}-\mathrm{H}_{2}\right)=3.24 \AA\right)$.

The $a b$ initio energies were used to parametrize the force field describing interaction energy between $\mathrm{H}_{2}$ and the pore walls. The distribution of adsorption energy in pores was then calculated for various boron contents and the pore width. The resulting 3D energy grids were implemented in GCMC code to perform large scale simulation of hydrogen adsorption isotherms. In what follows, we show that the substitution of just $5 \%$ of carbons by boron atoms almost doubles the strength of hydrogen interaction with the substituted pore wall and may bring the storage capacity of substituted carbon close to the limits required for practical applications.

It has been previously suggested that substituting carbon by boron causes a local distortion of the perfectly planar structure of graphite. ${ }^{10}$ No such deviation from planarity has been observed in the present $a b$ intio calculations. Hence, in the present work we modeled the substituted pore walls as flat sheets of graphenelike structures. The hydrogen molecules have been modeled by structureless superatoms interacting via Lennard-Jones (LJ) (6-12) potential. The interaction hydrogen-adsorbing surface was calculated assuming the additive LJ-type interaction of $\mathrm{H}_{2}$ super atom with all atoms of the pore wall within a cutoff distance of $15 \AA$. The $\varepsilon$ and $\sigma$ parameters of the $\mathrm{LJ}$ potential were adjusted in such a way that the energy experienced by $\mathrm{H}_{2}$ over the surface agrees with the values determined by $a b$ initio calculations. This required modification not only of $\mathrm{B}-\mathrm{H}_{2}$ interaction parameters, but also of those of the nearest and next-nearest neighboring carbons ( $\alpha$ and $\beta$ carbons). For $\mathrm{H}_{2}$ interactions with carbons more distant from boron usual values of $\mathrm{LJ}$ parameters have been used. ${ }^{20}$ Feynman-Hibbs quantum corrections $^{21-23}$ were applied to all interactions.

Figure 1 shows a map of minimum energy for $\mathrm{H}_{2}$ adsorption over an infinite graphene surface with single boron atom substituted at the $(0,0)$ position (left panel). The map was calculated as follow: a probe $\mathrm{H}_{2}$ superatom was placed at $(x, y, z)$ point over the substrate and the minimum of interaction energy $E_{\min }(x, y)$ has been found by varying probe's $z$ coordinate.

Two major conclusions come out from the analysis of $E_{\min }(x, y)$ energy landscape. First, the substitutional boron is not actually the location of strongest energy of adsorption,
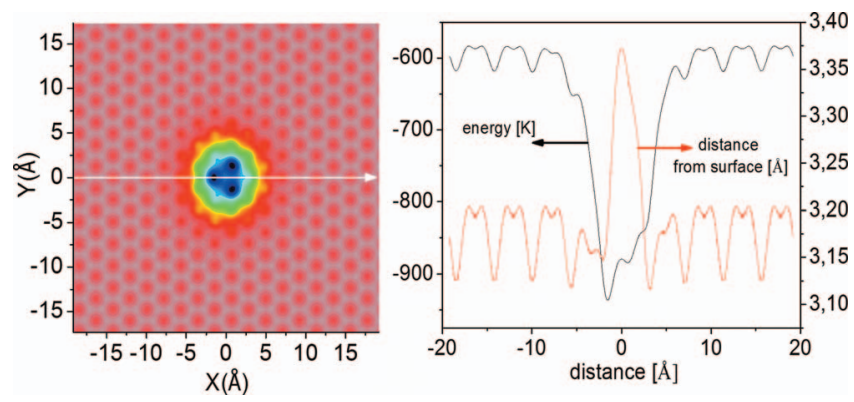

FIG. 1. (Left panel) Energy landscape of graphite with central carbon substituted by boron. Isoenergetic lines are separated by $\Delta E / k_{B}=10 \mathrm{~K}$ and go from $E / k_{B}=-580 \mathrm{~K}$ (pink gray) to $-940 \mathrm{~K}$ (navy). (right panel) Energy of adsorption $E_{\min }$ and corresponding distance from the substrate $z_{\min }$, along the $(x, 0)$ line.

but it contributes to a dramatic enhancement of the adsorption over the neighboring hexagons of the graphite lattice (in slightly off-center position). As the boron atom is larger than carbon $\left(\sigma_{\mathrm{B}}=3.54 \AA\right.$ versus $\left.\sigma_{\mathrm{C}}=3.34 \AA\right)$, there is a local weak maximum of the energy at the boron, and it is located farther from the surface (Fig. 1, right panel). Second, modification of the energy landscape extends far beyond the $\mathrm{C}_{\beta}$ carbons and is still perceptible $\sim 7 \AA$ from the substituted boron. It suggests that the adsorption properties of the whole graphene-based surface may be strongly modified by even small amounts of substitutional boron atoms.

To explore this possibility, we prepared graphene surfaces containing $1 \%, 2.5 \%, 5 \%$, and $10 \%$ of boron. The sites to be substituted were chosen at random, but observing a separation of at least three bonds between them. This topological constraint allowed us to use the $a b$ initio results obtained for an isolated substituted boron in an additive way.

For low substitution ratio the strong adsorption regions around the boron atoms remain well separated (Fig. 2, left panel). However, already at substitution ratio of 5\% these regions significantly overlap and the average adsorption energy increases with respect to "pristine" graphite (from $E / k_{B} \sim 600 \mathrm{~K}$ to $E / k_{B} \sim 900 \mathrm{~K}$ ); the energy of the strongest adsorption site even doubles (Fig. 2, right panel). At a substitution ratio of $10 \%$ the strongest adsorption energy reaches the value of $E / k_{B} \sim 1400 \mathrm{~K}$. However, at $10 \%$ substitution, the assumption of additive contribution of each boron inter-

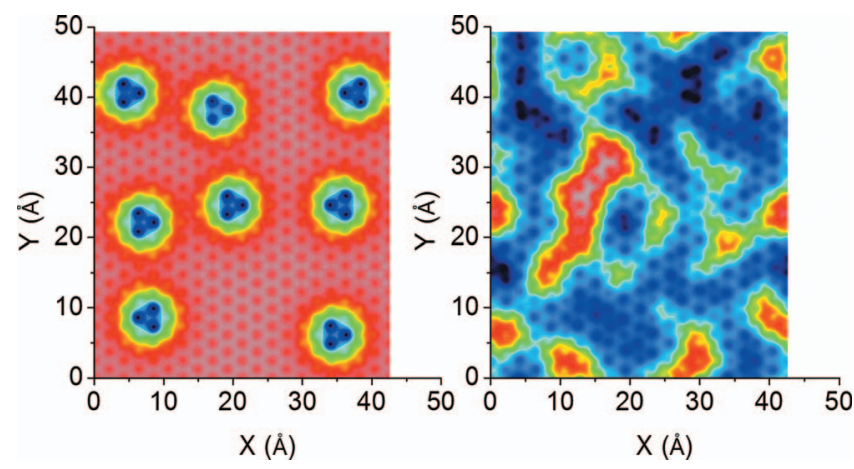

FIG. 2. Energy landscape of graphite surface containing 1\% (left) and 5\% (right) of substitutional boron atoms. Substitution is assumed to be random. Isoenergetic lines are separated by $\Delta E / k_{B}=10 \mathrm{~K}$; the color code on both graphs is identical to this figure: from $-580 \mathrm{~K}$ (pink gray) to $-1100 \mathrm{~K}$ (dark navy). 


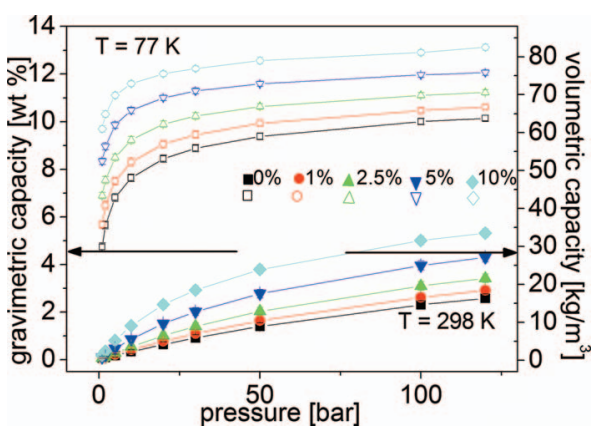

FIG. 3. Gravimetric (left axis) and volumetric (right axis) adsorption of hydrogen in graphene slit pores of width $d=1.2 \mathrm{~nm}$ as a function of pressure and the percentage of boron content in the pore wall, for $T=77$ and 298 K. For reference, the arrows indicate both gravimetric and volumetric U.S. DOE targets required to be reached by 2010 by the storing system.

action to the total adsorption energy is less certain: results should be revisited on the basis of ab initio calculations of $\mathrm{H}_{2}$ interaction with highly substituted graphene that are in progress.

Figure 3 shows the simulated isotherms of hydrogen adsorption (total amount stored), at $T=77$ and $298 \mathrm{~K}$ in pristine and substituted slit pores of $1.2 \mathrm{~nm}$ width. Such pores can accommodate up to three layers of hydrogen: two in direct contact with the pore walls and one between them. The low temperature adsorption results from the total filling of the contact layers and is already important $\left(\sim 60 \mathrm{~kg} / \mathrm{m}^{3}\right.$ at $P$ $=100$ bar) in pure carbon pores. The increase in the adsorption energy by substitution of $5 \%$ boron in the pore wall leads to additional pore filling (up to $\sim 80 \mathrm{~kg} / \mathrm{m}^{3}$ of hydrogen stored) which is possible mostly by stabilizing the third, middle, layer. At room temperature the results are even more dramatic: the amount stored in the boron-substituted pores doubles with respect to the pristine ones. This results in a material that compares favorably with both gravimetric and volumetric U.S. DOE application targets for 2010, though it must be noted that the targets requirement address the parameters of entire storing system, ${ }^{7}$ whereas our results represent merely the material storage capacity.

An equally important consideration for storage applications is the amount of hydrogen delivery in a complete adsorption-desorption cycle. Assuming conservatively that the system should operate purely by means of pressure sweeps (i.e., by varying the pressure between 1 and 100 bar), the delivery of stored hydrogen is almost complete $(\sim 97 \%)$ and independent of substitution ratio at room temperature. Therefore, the moderate wall heterogeneity introduced by substitution with boron is not detrimental for the potential room temperature applications of substituted nanoporous carbons. At $T=77 \mathrm{~K}$, although the total amount stored is significantly larger, only $\sim 53 \%$ can be recovered from the pure carbon pores and $\sim 25 \%$ from the pores substituted with $10 \%$ boron. This result agrees with theoretical considerations showing that the wall heterogeneity in general and the presence of strongly attractive adsorption sites in particular hinders the reversibility of adsorption in nanopores, especially at low temperatures. ${ }^{5}$ For comparison, if the explicit atomic structure of the pore wall is replaced by a structureless (smooth) surface, the corresponding deliveries (at $T=77 \mathrm{~K}$ ) are $\sim 65 \%$ from the pristine graphene pores and $\sim 30 \%$ form pores showing the same average adsorption energy as the pores containing $10 \%$ of boron. ${ }^{24}$

Since the Møller-Plesset approach slightly overestimates the adsorption energy and the real adsorbent is more heterogeneous than the modeled one, the calculated values define upper limits of the adsorption that could be observed in real samples. However, even in a worst case scenario, if our $a b$ initio calculations overestimated the adsorption energy by $10 \%-15 \%$ (it is likely much less), ${ }^{15}$ the adsorption reported for the sample with $10 \%$ boron would be closer to the one for $5 \%$ boron but still would show a significant improvement with respect to pure carbon.

Our calculations show, at least theoretically, nanoporous carbons partially substituted with boron can fulfill the requirements for practical use as hydrogen storage media. To the best of our knowledge, to date, carbons partially substituted with boron are the only candidates that can achieve the high storage without appealing to metal doping of the carbon backbone. The high capacity is not related to any particular crystallographic structure of the substituted pore wall but depends only on the B:C substitution ratio. Substituting only $5 \%-10 \%$ of carbons by boron atoms allows one to approach both gravimetric and volumetric directives of DOE with almost $100 \%$ delivery by pressure sweep. Obviously, experimental confirmation of our calculations is needed for validation of this theoretical conclusion.

Simple thermodynamics considerations ${ }^{5}$ previously demonstrated that $\sim 15 \mathrm{~kJ} / \mathrm{mol}$ is the optimum adsorption energy for $\mathrm{H}_{2}$ storage at room temperature in homogeneous materials. Our calculations show that boron doping of carbon results in energies that are close to optimum at low coverage ( $\sim 10.0-13.5 \mathrm{~kJ} / \mathrm{mol}$ for $5 \%-10 \%$ boron concentration), but decrease at high coverage $(\sim 5.0-6.0 \mathrm{~kJ} / \mathrm{mol})$, with an average of $7.5-10 \mathrm{~kJ} / \mathrm{mol}$ over a $0-100$ bar pressure cycle. Higher boron concentrations will be necessary to reach the optimum adsorption energy over a broad range of coverage. This analysis cannot be accomplished within the presented approach and is currently a subject of investigation.

This material is based on work supported in part by the Department of Energy under Award Nos. DE-FG0207ER46411 and DE-FC36-08GO18142 (L.F., B.K., P.F., and C.W.). We acknowledge the Wroclaw and Poznan Supercomputing and Networking Centers and University of Missouri Bioinformatics Consortium for the use of their computational facilities.

${ }^{1}$ S. Satyapal, J. Petrovic, C. Read, G. Thomas, and G. Ordaz, Catal. Today 120, 246 (2007).

${ }^{2}$ L. Schlapbach and A. Zuttel, Nature (London) 414, 353 (2001).

${ }^{3}$ L. Zubizarreta, E. I. Gomez, A. Arenillas, C. O. Ania, J. B. Parra, and J. J. Pis, Adsorption 14, 557 (2008).

${ }^{4}$ P. Benard and R. Chahine, Scr. Mater. 56, 803 (2007).

${ }^{5}$ S. K. Bhatia and A. L. Myers, Langmuir 22, 1688 (2006).

${ }^{6}$ J. Burress, M. Kraus, M. Beckner, R. Cepel, G. Suppes, C. Wexler, and P. Pfeifer, Nanotechnology 20, 204026 (2009).

${ }^{7} \mathrm{http}: / /$ www1.eere.energy.gov/hydrogenandfuelcells/mypp/pdfs/storage.pdf ${ }^{8}$ P. Pfeifer, J. W. Burress, M. B. Wood, C. M. Lapilli, S. A. Barker, J. S. Pobst, R. J. Cepel, C. Wexler, P. S. Shah, M. J. Gordon, G. J. Suppes, S. P. Buckley, D. J. Radke, J. Ilavsky, A. C. Dillon, P. A. Parilla, M. Benham, and M. W. Roth, Mater. Res. Soc. Symp. Proc. 1041, R02-02 
(2008).

${ }^{9}$ C. Kim, T. Fujino, T. Hayashi, M. Endo, and M. S. Dresselhaus, J. Electrochem. Soc. 147, 1265 (2000).

${ }^{10}$ M. Endo, T. Hayashi, S.-H. Hong, T. Enoki, and M. S. Dresselhaus, J. Appl. Phys. 90, 5670 (2001).

${ }^{11}$ Y.-H. Kim, Y. Zhao, A. Williamson, M. J. Heben, and S. B. Zhang, Phys. Rev. Lett. 96, 016102 (2006).

${ }^{12}$ Y. Ferro, F. Marinelli, A. Jelea, and A. Allouche, J. Chem. Phys. 120, 11882 (2004).

${ }^{13}$ C. Møller and M. S. Plesset, Phys. Rev. 46, 618 (1934).

${ }^{14}$ J. A. Pople, M. Head-Gordon, and K. Raghavachari, J. Chem. Phys. 87, 5968 (1987)

${ }^{15}$ R. C. Lochan and M. Head-Gordon, Phys. Chem. Chem. Phys. 8, 1357 (2006).
${ }^{16}$ S. Patchkovskii, J. S. Tse, S. N. Yurchenko, L. Zhechkov, T. Heine, and G. Seifert, Proc. Natl. Acad. Sci. U.S.A. 102, 10439 (2005).

${ }^{17}$ W. J. Stevens, H. Basch, and M. Krauss, J. Chem. Phys. 81, 6026 (1984).

${ }^{18}$ R. Krishnan, J. S. Binkley, R. Seeger, and J. A. Pople, J. Chem. Phys. 72, 650 (1980).

${ }^{19}$ M. J. Frisch, G. W. Trucks, H. B. Schlegel et al., Gaussian03, Revision C.02, Gaussian, Inc., Wallingford, CT, 2004.

${ }^{20}$ V. Buch, J. Chem. Phys. 100, 7610 (1994).

${ }^{21}$ Q. Wang and J. K. Johnson, J. Phys. Chem. B 103, 277 (1999).

${ }^{22}$ P. Kowalczyk, H. Tanaka, R. Hoyst, K. Kaneko, T. Ohmori, and J. Miyamoto, J. Phys. Chem. B 109, 17174 (2005).

${ }^{23}$ V. A. Kumar, H. Jobic, and S. K. Bhatia, Adsorption 13, 501 (2007).

${ }^{24}$ B. Kuchta, L. Firlej, P. Pfeifer, and C. Wexler, Carbon 48, 223 (2010). 